\title{
CD9 tetraspanins convey robustness to CXCR4b signalling during collective cell migration
}

\author{
Marsay KS ${ }^{1,3,4}$, Greaves $\mathrm{S}^{2}$, Roehl $\mathrm{H}^{2}$, Monk PN ${ }^{3}$, Carney T. J., ${ }^{4,5}$ and Partridge $\mathrm{L}^{1}$ \\ ${ }^{1}$ Department of Molecular Biology and Biotechnology, University of Sheffield, Sheffield UK \\ ${ }^{2}$ Department of Biomedical Science University of Sheffield, Sheffield UK \\ ${ }^{3}$ Department of Infection, Immunity and Cardiovascular Science, University of Sheffield, \\ Sheffield UK. \\ ${ }^{4}$ Institute of Molecular and Cell Biology (IMCB), A*STAR (Agency for Science, Technology and \\ Research), 61 Biopolis Drive, 138673, Singapore \\ ${ }^{5}$ Lee Kong Chian School of Medicine, Experimental Medicine Building, Yunnan Garden \\ Campus, 59 Nanyang Drive, Nanyang Technological University, 636921, Singapore
}

\begin{abstract}
Collective cell migration is essential for embryonic development and homeostatic processes. During zebrafish development, the posterior lateral line primordium ( $p L L P$ ) navigates along the embryo flank by collective cell migration. The chemokine receptors, CXCR4b and CXCR7b, as well as their cognate ligand, CXCL12a, are essential for this process. Knockdown of the zebrafish CD9 tetraspanin orthologue, $c d 9 b$, displayed mild pLL abnormalities. Through generation of CRISPR mutants, we show that $c d 9 a$ and $c d 9 b$ function partially redundantly in pLLP migration, which is delayed in the $c d 9 b$ single and $c d 9 a ; c d 9 b$ double mutants. This delay led to a transient reduction in neuromast numbers. Loss of both CD9a and CD9b sensitized embryos to reduced CXCR4b and CXCL12a levels. Together these results provide evidence that CD9 modulates collective cell migration of the pLLP through promoting CXCR4b signalling.
\end{abstract}

\section{Introduction}

Cells can migrate individually or in groups, the latter is known as collective cell migration. During this process, cells exhibit coordinated behaviour, group polarisation and maintain cellcell contacts (Olson \& Nechiporuk, 2018). This mode of migration is employed during embryonic development in the morphogenesis of multiple organ systems, whilst collective cell migration is important for effective immune responses. This mechanism contributes to several diseases including metastatic cancer and rheumatoid arthritis (Gallardo et al., 2010).

The zebrafish lateral line is a series of mechanosensory organs (neuromasts), which are distributed along the lateral surface of the zebrafish body and connected to the CNS by afferent axons (Dijkgraaf, 1962). This arrangement of innervated neuromasts is achieved by the collective migration of a placodal primordium of approximately 100 cells, arising just posterior to the otic placode (Metcalfe, Kimmel and Schabtach, 1985). This posterior lateral line primordium (pLLP), then migrates along the horizontal myoseptum to the tip of the tail. During migration, clusters of cells are deposited from the trailing end of the pLLP, which ultimately differentiate into neuromasts. 
Primordium migration is directed by expression of the chemokine CXCL12a along the horizontal myoseptum, which is received by two chemokine receptors expressed within the primordium (Haas and Gilmour, 2006; Dambly-Chaudière, Cubedo and Ghysen, 2007; Valentin, Haas and Gilmour, 2007). CXCR4b is expressed in the leading two thirds of the primordium, where ligand binding induces $G \beta 1$ signalling and actin polymerisation to promote a migratory phenotype (Xu et al., 2014). CXCR7 is expressed in the anterior-most third and functions as a ligand sink, allowing the formation of a local CXCL12a gradient. This results in directed migration of the primordium along the CXCL12a pathway towards the caudal fin (Donà et al., 2013; Venkiteswaran et al., 2014).

There is evidence that the membrane environment of chemokine receptors, including CXCR4 and CXCR7, strongly influences their signalling properties. This includes homodimerisation, oligomerisation and heteromerisation with other membrane receptors (Stephens and Handel, 2013). In addition, chemokine receptors have been linked to membrane microdomains where the lipid environment strongly modulates function (Thelen and Legler, 2018).

Tetraspanins are a large family of small integral membrane proteins, which have been shown to organise neighbouring membrane proteins into complexes called tetraspanin enriched microdomains (TEMs). This is often referred to as the tetraspanin web, as different interactions build to form dynamic signalling networks that often induce similar functional outcomes (Tarrant et al., 2003). Thus, tetraspanins are associated with a wide variety of cellular functions including signalling and cell migration (Yáñez-Mó et al., 2009) . For example, the tetraspanin CD9 is downregulated in many human cancers including lung, breast and ovarian, and reduced CD9 expression is related to colon cancer metastasis (Charrin et al., 2003). In particular, CD9 has been shown to regulate, and be regulated by, CXCL12-CXCR4 signalling (Leung et al., 2011; Arnaud et al., 2015; Brzoska et al., 2015). CD9 was found in close proximity to CXCR4 on the membrane of B acute lymphoblastic leukaemia cells in vitro, and enhanced their CXCL12 dependent migration (Arnaud et al., 2015).

If regulation of CXCL12/CXCR4 signalling by CD9 is conserved, we hypothesised CD9 would be strongly expressed in the zebrafish pLLP, where it might modulate CXCR4 signalling and thus pLLP migration. Indeed microarray experiments have localised the zebrafish $c d 9 b$ paralogue to the migrating primordium, whilst a morpholino experiment indicated loss of $c d 9 b$ altered primordium structure at $36 \mathrm{hpf}$ and reduced neuromast number at $48 \mathrm{hpf}$ (Gallardo et al., 2010).

In this study we aimed to investigate further the role of zebrafish CD9 orthologues in CXCR4bmediated pLLP migration, through use of genetic knockouts. We show expression of both $c d 9 a$ and $c d 9 b$ paralogues in the primordium. As the $c d 9 b$ mutants failed to fully replicate $c d 9 b$ morphant pLL phenotypes, we generated $c d 9 a ; c d 9 b$ double knock outs ( $c d 9$ dKOs). These showed both reduced migration of the pLLP and increased sensitivity to reduced levels of CXCR4b and CXCL12a, supporting a role for CD9 in regulation of CXCR4 signalling. 


\section{Methods}

\section{Zebrafish Maintenance}

Adult zebrafish (AB), $T g(-8.0 c / d n b: L Y-E G F P)^{2 f 106}$ and $c d 9 a / b$ mutants were housed and bred in a regulated 14:10 hour light: dark cycle under UK Home Office project licence 40/3459 in the Bateson Centre aquaria at the University of Sheffield or in the Singapore IMCB zebrafish facility under the Biological Resource Centre oversight with project license IACUC 140924. Zebrafish were raised under the standard conditions at $28^{\circ} \mathrm{C}$ (Nusslein-Volhard, 2002). Ages are expressed as hours (hpf) or days (dpf) postfertilisation.

\section{Morpholino Injection}

Antisense morpholino oligonucleotides (MO) were obtained from GeneTools LLC and resuspended in MilliQ $\mathrm{H}_{2} \mathrm{O}$ to give a stock concentration of $1 \mathrm{mM}$ and injected in one-cell stage embryos. A Flaming/Brown micropipette puller was used to create micro-injection needles from borosilicate glass capillary tubes $(0.5 \mathrm{~mm}$ inner diameter, Sutter). The PV800 Pneumatic PicoPump, as part of the micro-injection jig, was set up to release the required amount of injection material by adjusting the air pressure and air expulsion time. For the knockdown of $c d 9 b$, two MOs were designed, a translation blocker with the following sequence (cd9b MO1): $5^{\prime}$-tttatgaggagaaacccaagactga-3' and a splice site blocker ( $c d 9 b$ i2e3) with the following sequence was used: $5^{\prime}$-aacccctgaacacagagaaacaaca- $3^{\prime}$, whilst the published mismatch MO was used 5'- tttccctgctgcttatacagcgatg - $3^{\prime}$ (Trikic et al., 2011). For knockdown of $c x c r 4 b$ and $s d f 1 a$, the following sequences were used respectively, $5^{\prime}$-aatgatgctatcgtaaaattccat-3'and 5'-ttgagatccatgtttgcagtgtgaa-3' (David et al., 2002).

\section{Zebrafish mutant production} $c d 9 b$ mutants were created from $A B$ embryos using transcription activator-like effector nucleases (TALEN) and maintained on an AB background. TALEN (ZGene Biotech Inc., Taiwan) were provided in a pZGB4L vector, targeting the $c d 9 b$ sequence $5^{\prime}$-ttgctctttatcttca- $3^{\prime}$. Injected embryos were outcrossed and sequenced to identify mutations. A frameshifting deletion mutation (c.42_49del) was selected that caused premature termination within the first transmembrane domain ( $c d 9 b^{p g 15}$ allele). CRISPR-Cas9 was used to create $c d 9 a$ mutants, using the gRNA sequence 5'-gagtgtatatcctcattgcgg-3' targeting the 3rd exon encoding part of the second transmembrane domain. $c d 9$ dKO mutants were created by injecting the above $c d 9 a$ gRNA and Cas9 RNA into $c d 9 b^{p 915}$ embryos. These fish were screened for germline transmission by sequencing and backcrossed to $c d 9 b^{p g 15}$ mutants. An indel mutation deleting $4 \mathrm{bp}$ and inserting 8bp (c.180_187delinsTCGCTATTGTAT; $c d 9 a^{l a 61}$ ) generated a frameshift mutation resulting in a premature stop codon in exon 3 , which was predicted to truncate the protein before the large extracellular domain. Heterozygous fish of the same genotype were incrossed and adult F2 fish were genotyped to identify homozygous $c d 9 b^{p g 15}$; $c d 9 a^{1 a 61}$ ( $c d 9$ dKO).

\section{In situ hybridisation}

Embryos were raised at $28^{\circ} \mathrm{C}$ in petri dishes containing E3 solution. The E3 was changed daily, and any dead embryos removed. At 30-32 hpf, embryos were anaesthetised using tricaine, dechorionated and then fixed overnight at $4^{\circ} \mathrm{C}$ using $4 \%(\mathrm{w} / \mathrm{v})$ paraformaldehyde (PFA; Sigma-Aldrich, UK) in PBS. Embryos were washed in PBS/0.05 \% (v/v) Tween 20 (PBST), then put through a $\mathrm{MeOH} / \mathrm{PBS}$ series using $30 \%, 60 \%$ and $100 \%$ (v/v) MeOH before being stored in $100 \% \mathrm{MeOH}$ (Sigma-Aldrich) at $-20^{\circ} \mathrm{C}$. In situ hybridisation was carried out as described by Thisse \& Thisse, 2008, except for the embryo digestion with proteinase K, for which 30-32 hpf embryos were digested with $10 \mathrm{mg} / \mathrm{ml}$ proteinase $\mathrm{K}$ at $20^{\circ} \mathrm{C}$ for 22 min. Primers used for PCR generation of the in situ probes are given in table 1 below. The protocol was conducted with 
the embryos in $1.5 \mathrm{ml}$ microfuge tubes for the first two days, after which they were held in 12-well plates for staining before transferring back to microfuge tubes for storage. Stained embryos were stored in the dark in $80 \%$ (v/v) glycerol.

\begin{tabular}{|l|l|l|}
\hline Probe & Primer name & Sequence $\left(5^{\prime}\right.$-3) \\
\hline Claudin b & Claudinb F & aaacgaaaaagcatggcatc \\
\hline Claudin b & Claudinb R & gaggctgtttcaaacgtggt \\
\hline CD9a & CD9a F & gtcatattcgcggtcgaagt \\
\hline CD9a & CD9a R & ctgcgagaacaacaaagcaa \\
\hline CD9b & CD9b F & gttcgccacaagtgcctgat \\
\hline CD9b & CD9b R & tacatgttactttctctccaaacaat \\
\hline
\end{tabular}

Table 1. Primers for making ISH probes

Time lapse imaging

Time-lapse recording was performed using an inverted Zeiss LSM700 Confocal microscope. All larvae were anaesthetised using $0.02 \%$ tricaine and then embedded on their side in $1 \%$ low melting agarose (Lonza) on a glass bottom dish (MalTEK Corporation) and covered with E3 supplemented with $0.02 \%$ tricaine. Separate Z-stack images covering the depth of the horizontal myoseptum were taken at specified intervals over a specified period and assembled into a final movie at a specified frame rate.

\section{Statistics}

Data distribution was first assessed for normality using a D'Agostino-Pearson omnibus K2 normality test on the experimental residuals, as well as creating a histogram of residuals. For normally distributed data, an ANOVA with Dunnet's or Holms-Sidak multiple comparisons tests were used. For non-normally distributed data non-parametric tests, the Mann-Whitney $\mathrm{U}$ test or Kruskal-Wallis with Dunn's multiple comparisons test, were used. 


\section{$\underline{\text { Results }}$}

Both $c d 9$ zebrafish paralogues are expressed in the lateral line Zebrafish possess two CD9 paralogues, CD9a [NP_997784] and CD9b [NP_998593] which show $60 \%$ and $59 \%$ identity to human CD9 respectively and 63\% identity to each other (MUSCLE) (Edgar, 2004). Whole mount in-situ hybridisation (WISH) using probes against $c d 9 a$ and $c d 9 b$ demonstrated expression of both paralogues in the migrating primordium at $30 \mathrm{hpf}$. Expression of both $c d 9 a$ and $c d 9 b$ was also observed in recently deposited neuromasts and was retained in neuromasts until at least $5 \mathrm{dpf}$ (Fig. 1A-F).
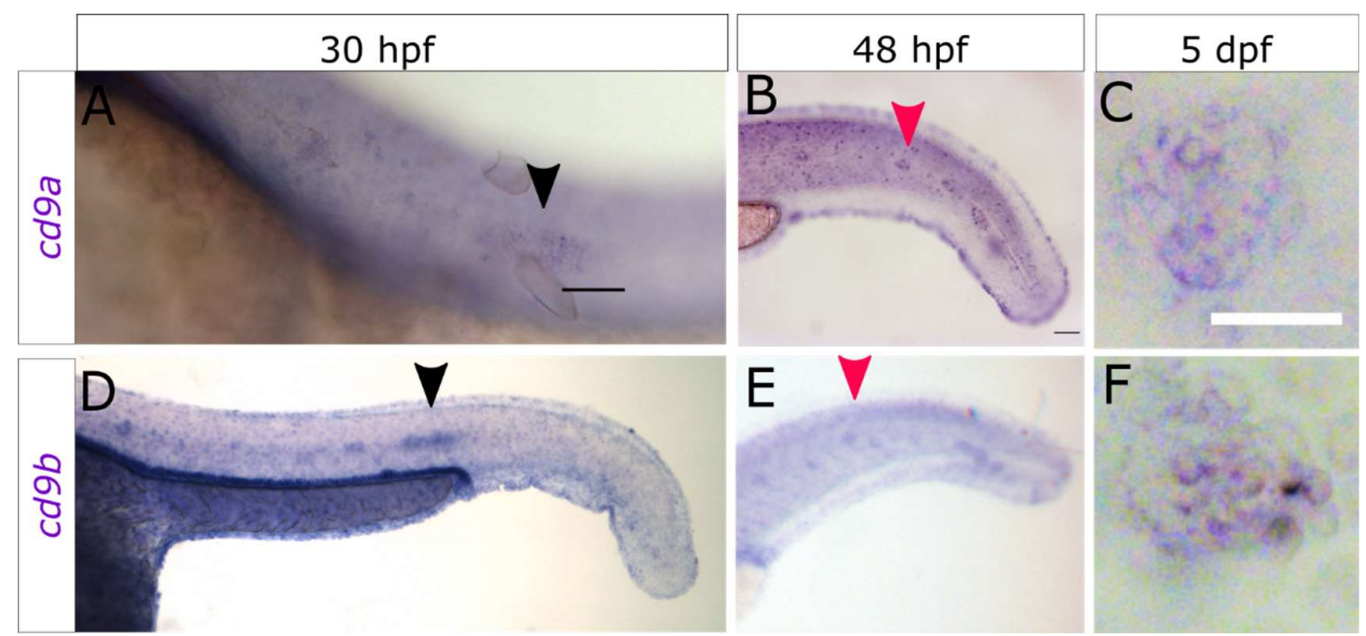

Figure 1. $c d 9 a$ and $c d 9 b$ are expressed in the pLLP during zebrafish development.

A-C: Micrographs of WISH of $c d 9 a$ at (a) $30 \mathrm{hpf}$ (b) $48 \mathrm{hpf}$ and (c) $5 \mathrm{dpf}$. D-F: WISH of $c d 9 b$ at (d) $30 \mathrm{hpf}$ (e) $48 \mathrm{hpf}$ and (f) $5 \mathrm{dpf}$. Arrows indicate primordium (black) and neuromasts (red). Scale bar indicates $50 \mu \mathrm{m}$ (black) and $100 \mu \mathrm{m}$ (white).

\section{CD9b morphant phenotype}

To evaluate if there was a role for either paralogue in $\mathrm{pLL}$ primordium migration, we initially focused on $c d 9 b$ as its expression in the primordium appeared stronger than $c d 9 a$ (compare Fig. 1A with 1D). Two different MOs were designed to target $c d 9 b ; c d 9 b \mathrm{MO} 1$ was a translation blocking $\mathrm{MO}$ which targeted the $5^{\prime}$ UTR of $c d 9 b$ RNA and $c d 9 b$ i2e3 was a splice blocking MO designed against the intron 2 - exon 3 splice site. These MOs were injected independently into 1-cell stage embryos to ensure that they produce the same phenotype.

Embryos injected with either CD9b MO1 or CD9b i2e3, but not the mismatch or uninjected embryos, showed a significant decrease in neuromasts deposited (Fig. 2H). For embryos injected with CD9b MO1, there was a decrease in the percentage of trunk length between the first and last neuromasts (Fig. 2I). This suggests that the primordium is stalling prematurely or migrating more slowly. This recapitulates and expands on the work by Gallardo et al., 2010 which suggested a role for CD9b in lateral line development. 

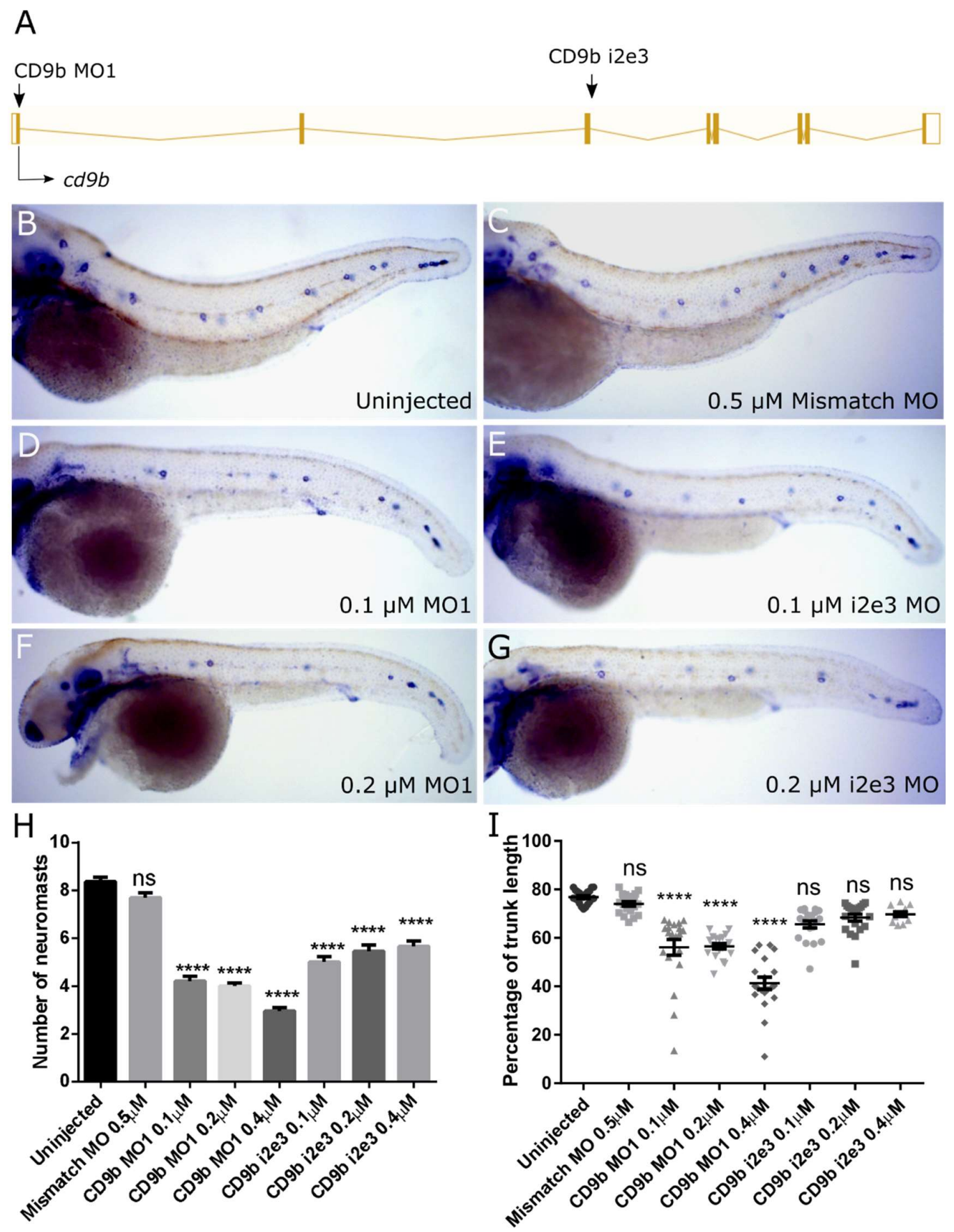

Figure 2. $c d 9 b \mathrm{MO}$ cause a decrease in neuromasts deposited at $52 \mathrm{hpf}$.

A: Intron exon diagram of the $c d 9 b$ gene with $\mathrm{MO}$ target sites. B-G: Representative images of WISH of claudin b at $52 \mathrm{hpf}$ of (b) uninjected embryos, (c) embryos with $0.5 \mu \mathrm{M}$ Mismatch, (d) $0.1 \mu \mathrm{M} c d 9 b \mathrm{MO1}$, (e) $0.1 \mu \mathrm{M} c d 9 b$ i2e3 MO1, (f) $0.2 \mu \mathrm{M} c d 9 b \mathrm{MO1}$, (g) $0.2 \mu \mathrm{M} c d 9 b$ i2e3 MO. H: Graph showing the number of neuromasts deposited at $52 \mathrm{hpf}$ is significantly decreased after injection with both $c d 9 b \mathrm{MO} 1$ and $c d 9 b$ i2e3 $\mathrm{MO}$, with as low as $0.1 \mu \mathrm{M} \mathrm{MO}$. I: Graph showing the percentage of trunk between the first and last deposited neuromast is decreased in $c d 9 b \mathrm{MO} 1$ morphants but not $c d 9 b$ i2e3 morphants. ANOVA with Dunnett's multiple comparisons test was used. $n=$ minimum 12 lateral lines analysed per condition. $* * * * p=<0.05$. 


\section{CD9b mutant phenotype}

To confirm this $\mathrm{MO}$ result, we created a TALEN mediated knockout of $c d 9 b$. A cd9b TALEN pair was designed by ZgeneBio using the program "TEL Effector Nucleotide Targeter 2.0". The TALEN pair was designed to target Exon 1 and predicted to cut in the 1st transmembrane domain (Fig. 3A). The TALEN pair was injected and after $72 \mathrm{hpf}$ a proportion of embryos were genotyped. Injected embryos showed clear mosaicism around the TALEN cut site so embryos were raised to create mosaic adult FOs. FOs transmitting a mutation to offspring were outcrossed with $A B$ fish and the resulting F1 offspring were raised. To create a cd9b homozygous mutant line with a single mutant allele, F1s with a c.42_49del mutation were selected, incrossed and the resulting offspring raised to adulthood. This allele $\left(c d 9 b^{p 915}\right)$ was used as it had the largest deletion and caused the earliest nonsense stop codon (Fig. 3B). The 8bp deletion in the $c d 9 b$ allele leads to a frameshift in exon 1, changing codon 15 from TTT (Phe) to CAA (Glu), then 22 aberrant amino acids followed by a stop codon (p.Phe15GlufsTer22).

$c d 9 b^{p g 15}$ homozygous mutants are viable and showed normal development. Loss of $c d 9 b$ in situ signal in the $c d 9 b$ mutants suggested strong nonsense mediated decay (NMD) of the mutated allele (Fig. S1). Due to the phenotypes seen in $c d 9 b$ morphants, it was expected that a lateral line phenotype would be seen in $c d 9 b$ mutants with fewer neuromasts deposited. However, no significant difference was found in the number of neuromasts at $52 \mathrm{hpf}$ (Fig. 3J). As $c d 9 b$ is expressed throughout pLL development, migration of the primordium as well as lateral line structure was assessed at $36 \mathrm{hpf}$. Although the $\mathrm{pLL}$ shows no structural abnormalities in $c d 9 b$ mutants at $52 \mathrm{hpf}$, it is possible that $c d 9 b$ mutants show a lateral line phenotype earlier in development and have recovered by $52 \mathrm{hpf}$. At $36 \mathrm{hpf}, c d 9 b$ mutants show delayed primordium migration, with the percentage of trunk traversed by the primordium reduced in mutants (Fig. 3E, G, I). 

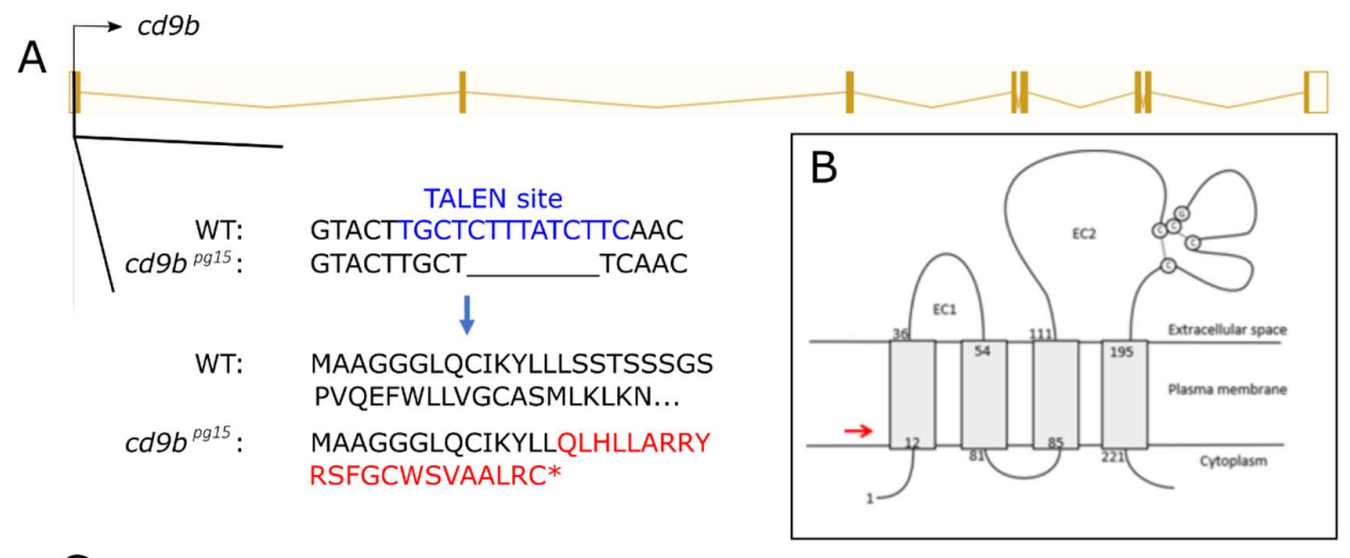

C WT

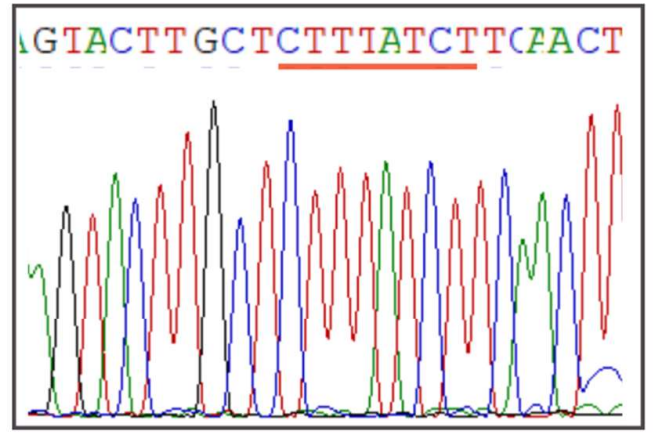

D $\quad c d 9 b^{p g 15}$
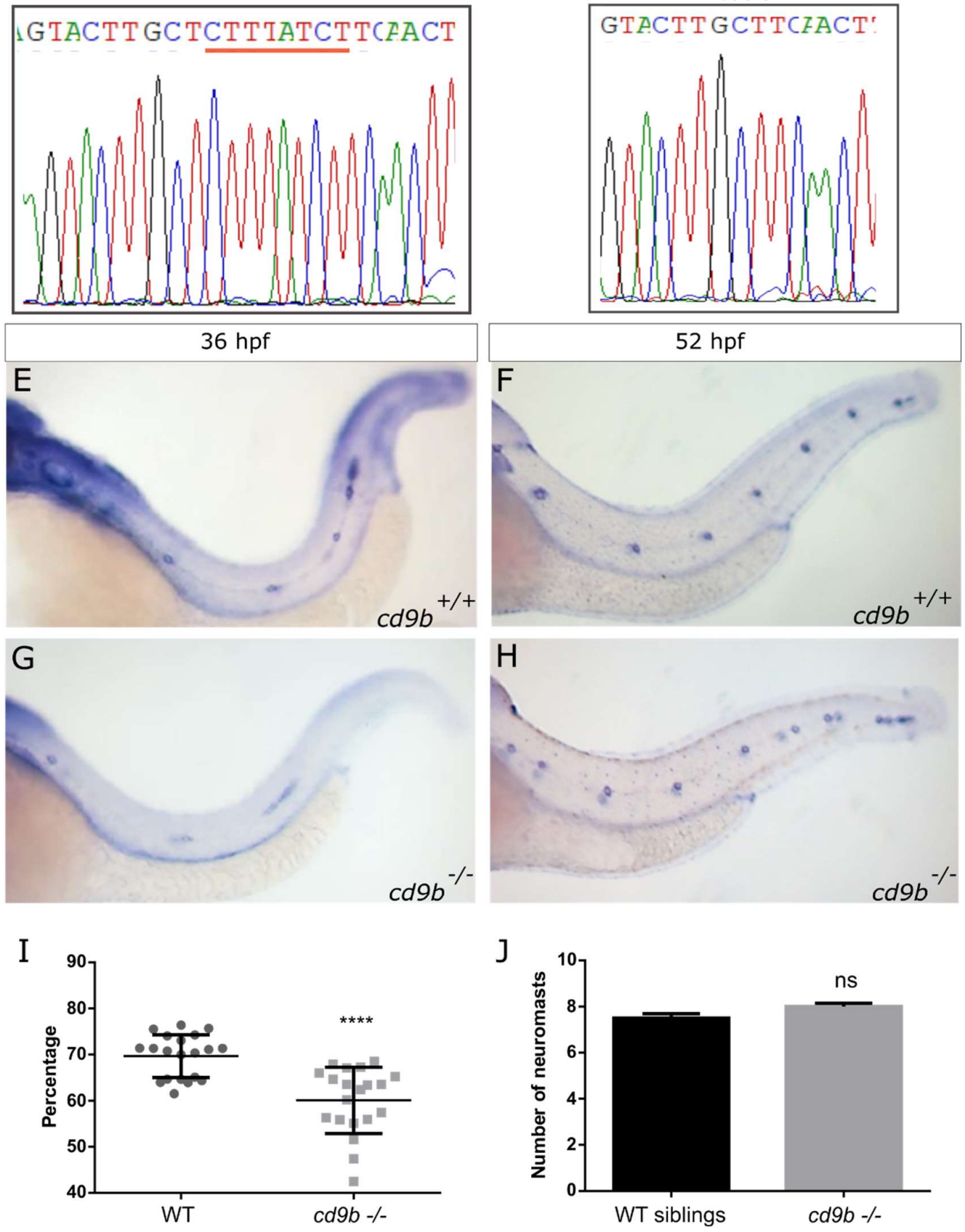
Figure 3. Generation of $c d 9 b$ mutant does not recapitulate morphant phenotype, although primordium migration is delayed at $36 \mathrm{hpf}$.

A: Nature of the $c d 9 b$ mutant allele showing TALEN site location within the intron-exon structure of the gene. Sequence of part of exon 1 shown below. The 8bp deletion in the $c d 9 b$ allele indicated under the WT sequence as dashes. This leads to a frameshift changing codon 15 from TTT (Phe) to CAA (Glu), then 22 aberrant amino acids (red lettering) followed by a stop codon $\left({ }^{*}\right)$. B: Schematic of the CD9b protein with location of premature stop codon given by red arrow. The disulfide bonds between the conserved CCG motif and conserved cysteines are indicated by the dashed lines. EC1/2= Extracellular domain 1/2, aa= amino acid. C-D: Sequence chromatograms of genomic DNA from (c) WT and (d) $c d 9 b^{p g 15}$ alleles. Deleted base pairs are underlined in red. E-H: Representative images of WISH of Claudin $b$ ISH on (e-f) wildtype and (g-h) $c d 9 b$ mutants at time shown. I-J: Graphs quantifying pLLP measurements in wild-type and $c d 9 b$ KOs; (i) the migration of the CD9b KO primordium at $36 \mathrm{hpf}$ is significantly delayed compared to wild-type. (j) There is no significance in number of neuromasts deposited at $52 \mathrm{hpf}$. Unpaired T test on untransformed data. $\mathrm{n}=$ minimum $20^{* * * *}=\mathrm{p}=<0.05$.

\section{Generation of a cd9a mutant}

To ascertain a role for CD9a, we targeted the cd9a gene using CRISPR/Cas9. A gRNA was designed to target $c d 9 a$ in exon 3 (Fig. 4A) and injected along with RNA encoding Cas9 into wild-type embryos to generate $c d 9 a$ knockouts. After screening for mosaicism, the CRISPR/Cas9 injected embryos were raised to maturity and screened for germline transmission of the $c d 9 a$ mutation.

F0s were outcrossed with wild-type fish to generate F1 cd9a single heterozygous mutants carrying an indel mutant allele (c.183_186delinsAT; $c d 9 a^{i c 62}$ ). This indel led to a frameshift mutation, changing codon 62 from ATT (Iso) to TGC (Cys), then generating 54 aberrant amino acids followed by a premature stop codon before the EC2 domain (p.Iso62Cysfs54Ter; Fig. 4). Heterozygous $c d 9 a^{+/ i c 62}$ adults were in-crossed to produce the F2 generation. Adult F2s were viable and fertile and were genotyped to identify homozygous $c d 9 a^{i c 62}$ knockouts. Loss of cd9a in situ signal in the $c d 9 a$ mutants suggested strong NMD of the mutated allele (Fig. S2). The effect of $c d 9 a \mathrm{KO}$ on $\mathrm{pLL}$ development was investigated but overall, there were no major defects. No significant difference was found in the number of neuromasts deposited at $48 \mathrm{hpf}$ or primordium migration at $36 \mathrm{hpf}$ (Fig. 4). 
bioRxiv preprint doi: https://doi.org/10.1101/2021.05.19.444012; this version posted May 20, 2021. The copyright holder for this preprint (which was not certified by peer review) is the author/funder, who has granted bioRxiv a license to display the preprint in perpetuity. It is made available under aCC-BY 4.0 International license.
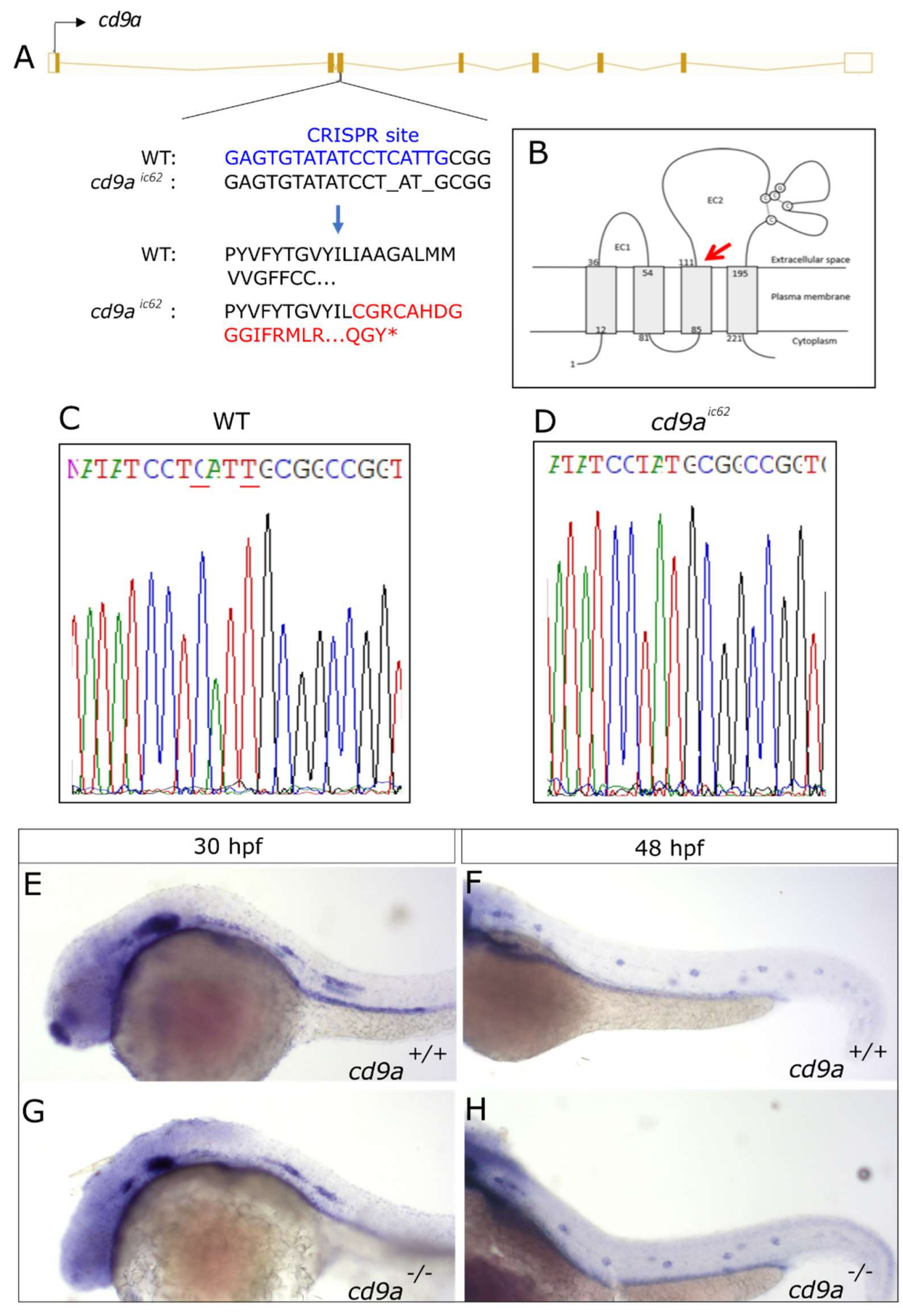

I Somite position of primordium at $36 \mathrm{HPF}$

了

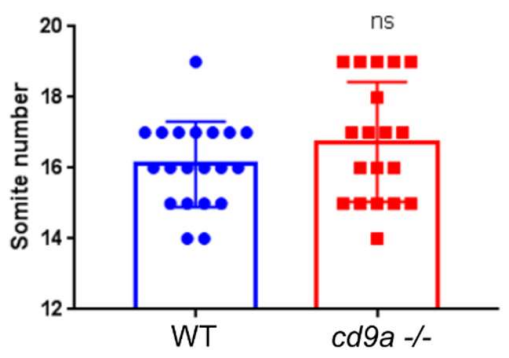

Neuromasts deposited at 48 HPF

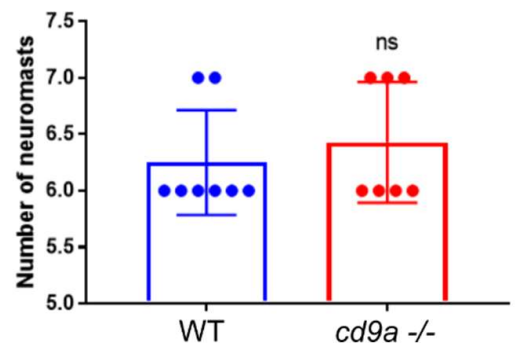


Figure 4. Generation of $c d 9 a$ mutant does not show any abnormal pLLP development. A: Nature of the $c d 9 a$ mutant allele showing CRISPR site location within the intron-exon structure of the gene. Sequence of part of exon 3 is shown below. The $2 \mathrm{bp}$ deletion in the $c d 9 a$ allele is indicated under the WT sequence as dashes. This leads to a frameshift changing codon 62 from ATT (Iso) to TGC (Cys), then 54 aberrant amino acids followed by a stop codon. B: Schematic of the CD9a protein with location of premature stop codon, indicated by red arrow, at a predicted 115 aa. EC1/2= Extracellular domain 1/2. C-D: Sequence chromatograms of genomic DNA from (c) WT and (d) $c d 9 a^{i c 62}$ allele. Deleted base pairs are underlined in red. E-F: claudin b expression in wild-type at (e) $30 \mathrm{hpf}$ and (f) $48 \mathrm{hpf}$. G-H: claudin b expression in CD9a mutants at (g) $30 \mathrm{hpf}$ and (h) $48 \mathrm{hpf}$. I-J: Graphs quantifying pLLP measurements in wildtype and CD9a KOs; (i) migration of the CD9a KO primordium at $36 \mathrm{hpf}$ is similar to wild-type. (j) There is no significance in number of neuromasts deposited. Significance was assessed using an unpaired t test. $\mathrm{N}=$ minimum 7 . Bars show mean $+/-\mathrm{SD}$.

\section{Generation of a $c d 9 a ; c d 9 b$ double mutants}

Due to the redundant nature of tetraspanins, and $c d 9 a$ having a similar mRNA expression pattern to $c d 9 b$, it was speculated that functional redundancy between CD9a and CD9b might be masking stronger phenotypes in the single mutants. Hence, we generated $c d 9 a ; c d 9 b$ double mutants.

To do this, the same cd9a gRNA as above (Fig. 4) and cas9 mRNA were injected into $c d 9 b^{p g 15}$ embryos. These fish were screened for mutation of $c d 9 a$ as above and then backcrossed to $c d 9 b^{p 15}$ mutants. An indel mutation in exon 3 was detected (c.180_184delinsTCGCTATTGTAT) that was predicted to lead to a frameshift mutation and an early stop codon, which truncated the protein before the EC2 domain (p.Leu61Alafs9Ter; Fig. 5). Transheterozygous fish were crossed and adult F2s were genotyped to identify doubly homozygous $c d 9 b^{p g 15}$; $c d 9 a^{l a 61}$ individuals, from now on referred to as $c d 9 \mathrm{dKOs}$. Adult $c d 9 \mathrm{dKOs}$ were viable and fertile and loss of $c d 9 a$ in situ signal in this $c d 9 a$ allele again suggested strong NMD of the mutated allele (Fig. S3). It was expected that with both paralogues knocked out, the lateral line phenotype would now be as severe as $c d 9 b$ morphants with fewer neuromasts deposited. However, no significant difference was found in the number of neuromasts at $48 \mathrm{hpf}$ (Fig. 5G). Primordium migration was significantly delayed in $c d 9$ dKO embryos at $36 \mathrm{hpf}$ (Fig. $5 \mathrm{H})$. This matches the phenotype shown by the $c d 9 b^{p g 15}$ single knockouts. To see if the knockout of $c d 9 a^{1 a 61}$ had an additional effect on the primordium delay, embryos from $c d 9 b^{p g 15}$ knockouts were directly compared to those from $c d 9$ dKOs (Fig. S4). The distance the primordium had migrated at 36 hpf was measured and no difference was seen between $c d 9 b^{p g 15}$ single KO and $c d 9 b \mathrm{dKO}$. 
A

C

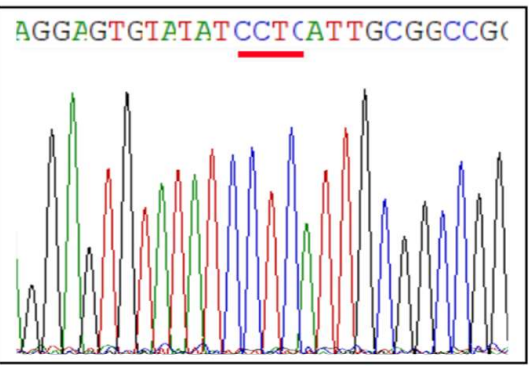

B

CRISPR site

WT: GAGTGTATATCCTCATTGCGG cd9a ${ }^{1 a 61}$ : GAGTGTATAT__tgcgtattgtatATTGCGG $\downarrow$

WT: PYVFYTGVYILIAAGALMM $c d 9 a^{1061}:$ PYVFYTGVYIAYCILRPVRS*

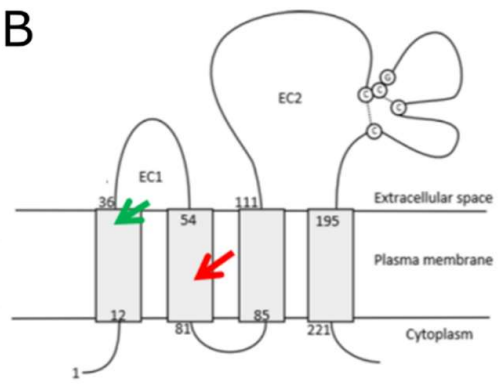

D $\quad c d 9 a^{\operatorname{lag1}}$

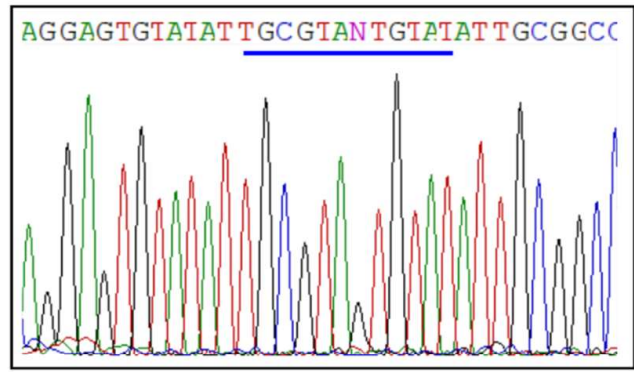

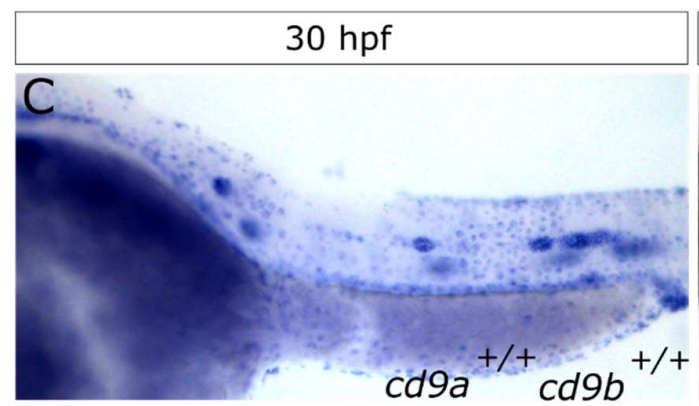

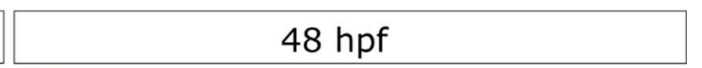

D

E

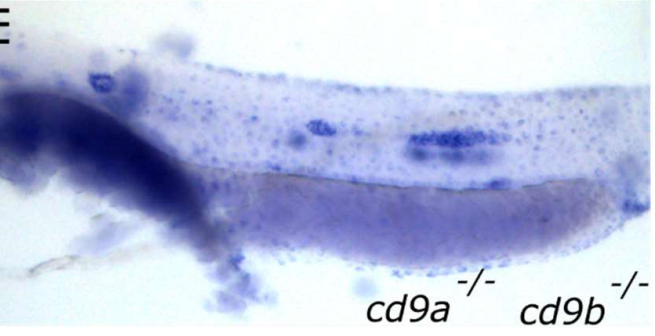

F

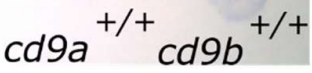

G

Somite position of primordium at $36 \mathrm{HPF}$

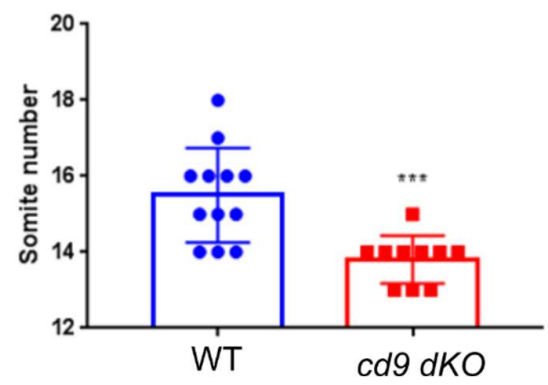

$\mathrm{H}$

Number of neuromasts deposited at $48 \mathrm{HPF}$

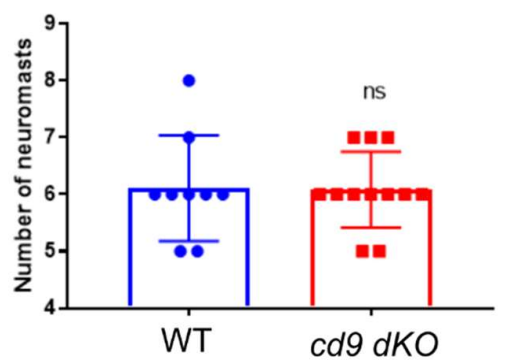

Figure 5. Generation of $c d 9 a$ mutant in the $c d 9 b^{p g 15} \mathrm{KO}$ background to create a double $c d 9$ KO mutant. 
bioRxiv preprint doi: https://doi.org/10.1101/2021.05.19.444012; this version posted May 20, 2021. The copyright holder for this preprint (which was not certified by peer review) is the author/funder, who has granted bioRxiv a license to display the preprint in perpetuity. It is made available under aCC-BY 4.0 International license.

A: cd9a mutant allele sequence from exon 3, showing CRISPR site location. The $4 \mathrm{bp}$ deletion (dashes) and 12bp insertion (lowercase) results in an 8bp insertion as indicated under the WT sequence. This leads to a frameshift changing codon 61 from CTC (Leu) to GCG (Ala), then 9 aberrant amino acids followed by a stop codon. B: Schematic of the CD9 protein with location of premature stop codon for CD9a (red arrow) and CD9b (green arrow) at a predicted 70 aa and 36 aa respectively. EC1/2= Extracellular domain 1/2. C-D: Sequence chromatograms of genomic DNA from (c) WT and (d) $c d 9 a^{l a 61}$ allele. Deleted base pairs are underlined in red and inserted base pairs in blue. E-F: claudin b expression in wild-type at (e) $30 \mathrm{hpf}$ and (f) $48 \mathrm{hpf}$. G-H: clnb expression in $c d 9$ dKO mutants at (g) $30 \mathrm{hpf}$ and (h) 48 hpf. I-J: Graphs showing pLLP measurements in wild-type and cd9 dKOs; (i) migration of the $c d 9 \mathrm{dKO}$ primordium at $36 \mathrm{hpf}$ is significantly delayed compared to wild-type. (j) There is no significance in number of neuromasts deposited. Significance was assessed using an unpaired t test. $N=$ minimum $9, p=$ $<0.001, \mathrm{n} 1=$ neuromast 1 etc. WT, wild type. Bars show mean +/- SD.

In order to further investigate the migration and organisation of the primordium in $c d 9 \mathrm{dKO}$ embryos they were crossed into the cldnb:gfp transgenic line. Under the cldnb promoter GFP is expressed in the lateral line primordium and newly deposited neuromasts (Haas and Gilmour, 2006). This allowed observation of the primordium migration in real time. Primordium migration appeared normal in the $c d 9$ dKO embryos (Fig. 6, Video S1, S2). At the leading edge of the primordium filopodia could be seen as well as proliferating cells. Within the migrating primordium rearrangements occurred and rosettes were formed, increasing the length of the primordium. As the trailing cells decelerated and deposited cells as neuromasts, the primordium reduced in size. Quantification of various aspects of the primordium during deposition revealed similar results in wild-type and mutants (Data not shown).
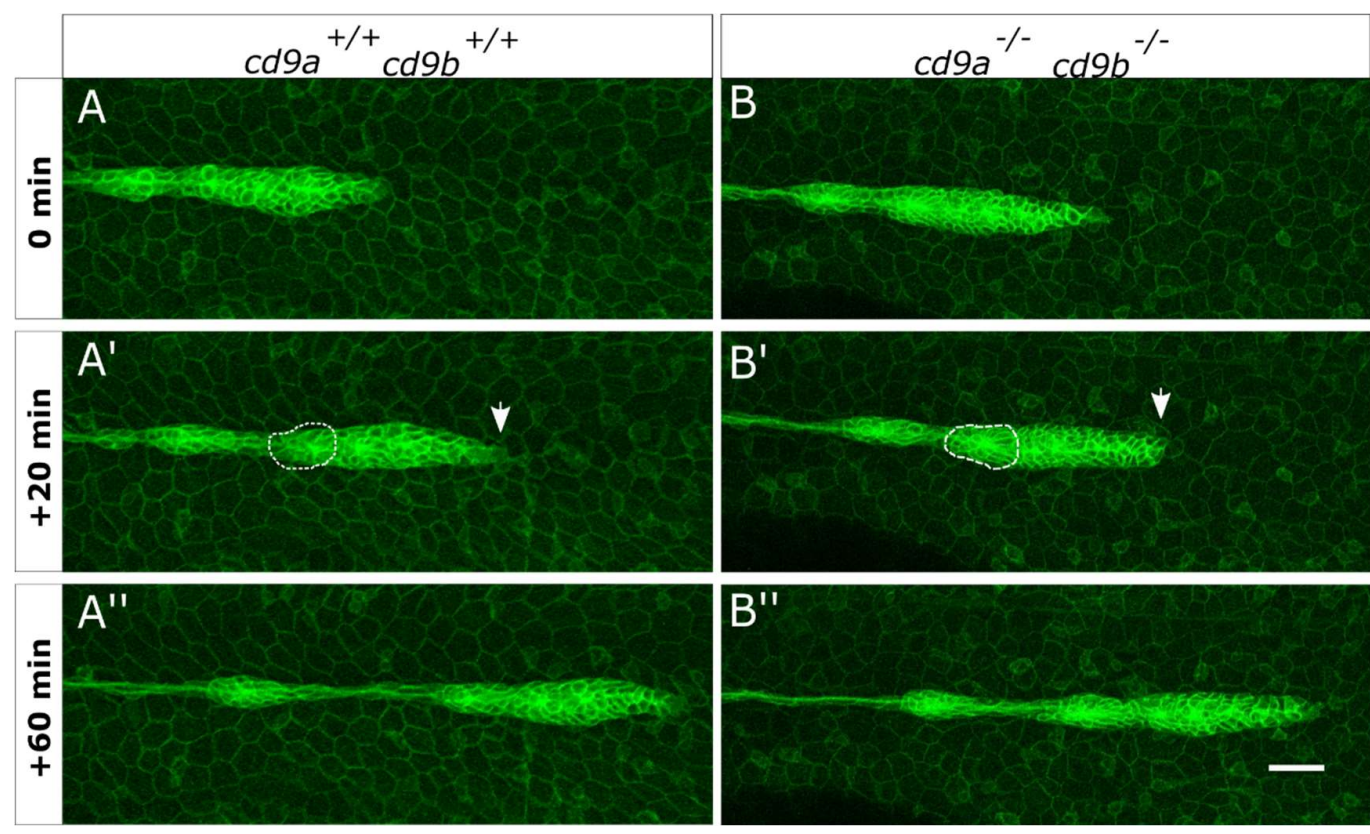

Figure 6. Primordium organisation appears normal in cd9 dKO(cldnb:gfp) embryos from 30 hpf.

A-B: Still images from a time-lapse recording of a migrating primordium in (a) wild-type and (b) $c d 9$ dKOs. 0 minutes shows initial deposition as a proneuromast becomes distinct from the primordium and then 2 sequential images show ( $\left.a^{\prime}-b^{\prime}\right) 20$ minutes and ( $\left.a^{\prime \prime}-b^{\prime \prime}\right) 60$ minutes later. In the primordium of both wild-type and $c d 9$ dKOs, filopodia can be seen at the leading 
edge (white arrow) and the formation of rosettes in the trailing edge (white dashed circle). Representative images from two videos that included two depositions each. Scale bar: $20 \mu \mathrm{m}$.

CD9 genetically interacts with the CXCR4b/CXCL12a pathway in the migrating zebrafish primordium

To investigate if CD9 was affecting migration in the zebrafish primordium through the CXCR4b/CXCL12a pathway, an experiment using morpholinos was conducted. CXCr4b and cxcl12a morpholinos induce premature stalling of the primordium at concentrations of 1.5 $\mathrm{mM}$ and $0.5 \mathrm{mM}$ respectively (David et al., 2002). $100 \mu \mathrm{M}$ of $c x c r 4 b$ morpholino was found to be the highest concentration injected into wild-type embryos that did not induce a phenotype. However, this concentration of morpholino resulted in a further delay of the primordium in the $c d 9$ dKOs (Fig. 7A). For $c x c / 12 a$ the highest concentration of morpholino that did not induce a phenotype in wild-type embryos was determined to be $12.5 \mu \mathrm{M}$ but this had no further effect on the primordium migration in $c d 9$ dKOs (data not shown). If the cxcl12a morpholino concentration was raised to $25 \mu \mathrm{M}$ then the primordium in both wildtype and $c d 9$ dKOs was delayed although the primordium in $c d 9$ dKOs was significantly more delayed (Fig. 7B).
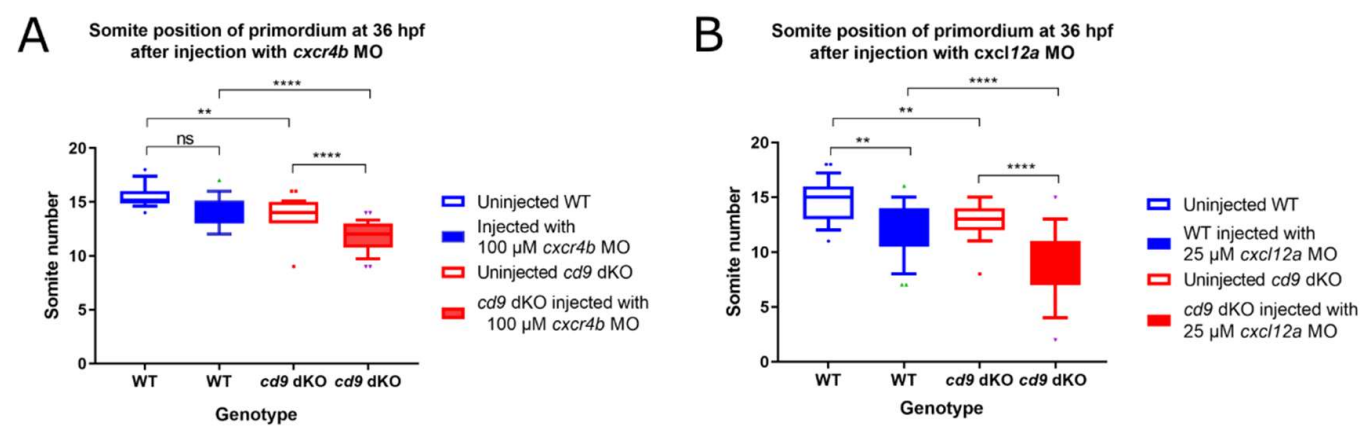

Figure 7. $c x c r 4 b$ and $c x c / 12 a$ MOs further delay primordium migration at $36 \mathrm{hpf}$ in the $c d 9$ dKO embryos.

A-B: Distance migrated by the primordium (labelled by cldnb in situ hybridisation) were recorded for (a) injection of $100 \mu \mathrm{M} c x c r 4 b \mathrm{MO}$, and (b) $25 \mu \mathrm{M} c x c / 12 a \mathrm{MO}$. Significance was assessed using one-way ANOVA, ${ }^{*}=p<0.05,{ }^{* *}=p<0.01,{ }^{* * *}=p<0.001 * * * *=p<0.0001 . \mathrm{N}=$ minimum 15. Box extends from the 25th to 75 th percentile and whiskers from 10 th to $90^{\text {th }}$.

\section{$\underline{\text { Discussion }}$}

In this study we investigated the role of CD9 in the migration of the posterior lateral line in zebrafish development. We first verified previous data showing $c d 9 b$ expression in the primordium at $30 \mathrm{hpf}$ (Gallardo et al., 2010). We also noticed $c d 9 b$ was expressed throughout recently deposited neuromasts at $48 \mathrm{hpf}$ and $5 \mathrm{dpf}$. Our MO knockdown recapitulated previous results from Gallardo et al., 2010. $c d 9 b$ morphants showed fewer neuromasts deposited, which suggested a role for CD9b in the development of the lateral line. A homozygous $c d 9 b$ mutant was created using TALENs but surprisingly, the morphant phenotype was not recapitulated in $c d 9 b$ homozygous mutants. This lack of phenotype could 
be due to several reasons. Firstly, the phenotype seen in $c d 9 b$ morphants could be due to off target effects of the morpholinos and not due to $c d 9 b$ knockdown. However, the fact that two different morpholinos and researchers induced the same phenotype suggests it was not the lack of specificity (Gallardo et al., 2010). Secondly, the CD9b truncated protein could have some residual function, although ISH results showed a downregulation of mRNA suggesting NMD)was occurring. Also, the $c d 9 b$ mutation aborts the normal sequence after aa15, before the EC2 domain, so any translated protein would be expected to have minimal function. Finally, the $c d 9 b$ morphant phenotypes may be specific but tetraspanin redundancy could rescue the phenotype in $c d 9 b$ mutants. Tetraspanins are well known for their redundancy within the tetraspanin family and mouse knockouts of single tetraspanins often appear healthy and viable with mild phenotypes, whereas double tetraspanin knockout mice often show increased numbers and severity of phenotypes (Knobeloch et al., 2000; Takeda et al., 2003, 2008; Wright et al., 2004; Hemler, 2005, 2008; Hassuna et al., 2009; Gartlan et al., 2010). In zebrafish, this redundancy may be amplified due to the occurrence of a fish-specific whole genome duplication in teleost fish. This means that many tetraspanins, for which there is one mammalian ortholog, have two paralogs in zebrafish (Meyer and Van De Peer, 2005; Brunet et al., 2006; Garcia-España et al., 2008; Huang et al., 2010; Howe et al., 2013). This is true for CD9, as mammals have a single CD9 whereas zebrafish have CD9a and CD9b. We demonstrated $c d 9 a$ to also be expressed in the $\mathrm{pLL}$ in a similar pattern to $c d 9 b$. Furthermore, NMD of mutated genes, as we have seen in both our mutants, has been shown to invoke an upregulation of closely related genes as a genetic compensation mechanism (El-Brolosy et al., 2019).

Following this, tetraspanin $c d 9 a$ was targeted for knockout using CRISPR technology. Cd9aic62 homozygous mutants and $c d 9 b^{p g 15} c d 9 a^{\text {la61 }}$ double homozygous mutants were generated. Cd9a knockout embryos displayed a normal lateral line phenotype with normal primordium migration and neuromast deposition. Initial analysis of $c d 9$ dKO embryos using the same measurements revealed that the number of neuromasts deposited and spacing were normal yet the distance of primordium migration at $36 \mathrm{hpf}$ was significantly delayed. It was expected that knockout of $c d 9 a$ would result in a stronger pLL phenotype but $c d 9$ dKOs showed a similar phenotype to the $c d 9 b$ KOs. Furthermore, when primordium migration of $c d 9 b$ single knockout was compared to that of the $c d 9$ dKOs there was no difference. These results suggest that the different phenotypes seen between the $c d 9 b$ morphants and mutants are not due to compensation by $c d 9 a$. It remains possible that there is compensation by another tetraspanin (Huang et al., 2005). Close relations to CD9 are the tetraspanins CD81 and CD63, which have been shown previously to substitute for CD9 in some circumstances.

Overexpression of CD81 partially rescued the infertility phenotype seen in CD9 KO mice (Kaji et al. 2002) and it was recently demonstrated that CD9 deletion in human melanoma cells was quickly compensated by CD63 expression upregulation (Suárez et al., 2021). It would be interesting to perform RNA-seq or SWATH-MS on the $c d 9$ dKOs to identify upregulated RNA and proteins respectively (Lin et al., 2019).

Tetraspanins contribute to cellular processes by organising molecules within the plasma membrane (Tarrant et al., 2003; Yáñez-Mó, Gutiérrez-López and Cabañas, 2011). CD9 is known to enhance chemokine signalling in several contexts. To test if this was relevant to chemokine signalling during zebrafish lateral line migration, sub-functional doses of either $c x c r 4 b$ or $c x c 112 a \mathrm{MO}$ were injected into wild-type or $c d 9$ dKO embryos. If there was some synergism between CD9 and CXCR4b/CXCL12a, it was theorised that a primordium lacking CD9 would be more susceptible to disruption than wild-type. Injection with a sub-functional concentration of $100 \mu \mathrm{M} c x c r 4 b \mathrm{MO}$ demonstrated that the $c d 9$ dKO larvae were more sensitive to $\mathrm{MO}$ treatment compared to wild-type with significant retardation in primordium 
migration in the dKO. This suggests that there is some interaction between CD9 and CXCR4b within the primordium that promotes migration. Injection with $c x c / 12 a \mathrm{MO}$ was more ambiguous as at a sub-functional concentration of $12.5 \mu \mathrm{M}$ neither the wild-type nor the $c d 9$ $\mathrm{dKO}$ primordium migration was perturbed. When the dose of $c x \mathrm{c} / 12 a$ morpholino was increased to $25 \mu \mathrm{M}$ a migratory delay was induced in both genotypes but the delay seen in the cd9 dKO primordium was significantly worse. Despite disruption in the presence of the $\mathrm{MO}$, the primordium was still able to eventually migrate the full length in the $c d 9 \mathrm{dKO}$ embryos, suggesting that CD9 is not essential for maintaining CXCR4b/CXCL12a signalling but may play a regulatory or buffering role. A further experiment to check for interaction between CD9 and CXCL12a/CXCR4b in the primordium would be to use immunohistochemistry to investigate co-localisation. Unfortunately, there are no tetraspanin, CXCL12a or CXCR4b antibodies available in zebrafish. However, two plasmids are available that encode $c x c r 4 b$-egfp or cxcl12a-venus constructs (Minina, Reichman-Fried and Raz, 2007). An interesting experiment would be to inject embryos with these plasmids and visualise the distribution of fluorescent versions of the proteins in the CD9 dKOs. We propose that, within the primordium, CD9b is functioning to compartmentalise CXCR4b to orchestrate and amplify signalling upon ligand binding but is not essential. This would explain why only subtle differences are seen between the wild-types and $c d 9$ dKOs.

\section{$\underline{\text { References }}$}

Arnaud, M.-P. et al. (2015) 'CD9, a key actor in the dissemination of lymphoblastic leukemia, modulating CXCR4-mediated migration via RAC1 signaling', Blood, 126(15), pp. 1802-1812. doi: 10.1182/blood-2015-02-628560.

Brunet, F. G. et al. (2006) 'Gene loss and evolutionary rates following whole-genome duplication in teleost fishes', Molecular Biology and Evolution. doi: 10.1093/molbev/msl049. Brzoska, E. et al. (2015) 'Sdf-1 (CXCL12) induces CD9 expression in stem cells engaged in muscle regeneration', Stem Cell Research and Therapy, 6(1), pp. 1-15. doi: 10.1186/s13287015-0041-1.

Charrin, S. et al. (2003) 'Multiple levels of interactions within the tetraspanin web', Biochemical and Biophysical Research Communications, 304(1), pp. 107-112. doi: 10.1016/S0006-291X(03)00545-X.

Dambly-Chaudière, C., Cubedo, N. and Ghysen, A. (2007) 'Control of cell migration in the development of the posterior lateral line: antagonistic interactions between the chemokine receptors CXCR4 and CXCR7/RDC1', BMC Developmental Biology, 7(1), p. 23. doi: 10.1186/1471-213X-7-23.

David, N. B. et al. (2002) 'Molecular basis of cell migration in the fish lateral line: role of the chemokine receptor CXCR4 and of its ligand, SDF1.', Proceedings of the National Academy of Sciences of the United States of America, 99(25), pp. 16297-16302. doi: 10.1073/pnas.252339399.

Dijkgraaf, B. Y. S. (1962) 'LATERAL-LINE ORGANS', pp. 51-105.

Donà, E. et al. (2013) 'Directional tissue migration through a self-generated chemokine gradient', Nature, 503(7475), pp. 285-289. doi: 10.1038/nature12635.

Edgar, R. C. (2004) 'MUSCLE: Multiple sequence alignment with high accuracy and high throughput', Nucleic Acids Research, 32(5), pp. 1792-1797. doi: 10.1093/nar/gkh340. El-Brolosy, M. A. et al. (2019) 'Genetic compensation triggered by mutant mRNA degradation Europe PMC Funders Group', Nature, 568(7751), pp. 193-197. doi: 10.1038/s41586-0191064-z.Genetic.

Gallardo, V. E. et al. (2010) 'Molecular dissection of the migrating posterior lateral line primordium during early development in zebrafish', BMC Developmental Biology, 10(1), p. 120. doi: 10.1186/1471-213X-10-120.

Garcia-España, A. et al. (2008) 'Appearance of new tetraspanin genes during vertebrate 
evolution', Genomics. doi: 10.1016/j.ygeno.2007.12.005.

Gartlan, K. H. et al. (2010) 'A Complementary Role for the Tetraspanins CD37 and Tssc6 in

Cellular Immunity', The Journal of Immunology. doi: 10.4049/jimmunol.0902867.

Haas, P. and Gilmour, D. (2006) 'Chemokine Signaling Mediates Self-Organizing Tissue

Migration in the Zebrafish Lateral Line Short Article', pp. 673-680. doi:

10.1016/j.devcel.2006.02.019.

Hassuna, N. et al. (2009) 'Strategies for Targeting Tetraspanin Proteins Potential Therapeutic Applications in Microbial Infections', 23(6), pp. 1-19.

Hemler, M. E. (2005) 'TETRASPANIN FUNCTIONS AND ASSOCIATED MICRODOMAINS', 6, pp. 801-811. doi: 10.1038/nrm1736.

Hemler, M. E. (2008) 'Targeting of tetraspanin proteins - potential benefits and strategies', 7(9), pp. 747-758. doi: 10.1038/nrd2659.Targeting.

Howe, D. G. et al. (2013) 'ZFIN, the Zebrafish Model Organism Database: increased support for mutants and transgenics.', Nucleic acids research, 41(Database issue), pp. D854-60. doi: 10.1093/nar/gks938.

Huang, S. et al. (2005) 'The phylogenetic analysis of tetraspanins projects the evolution of cell-cell interactions from unicellular to multicellular organisms', Genomics, 86(6), pp. 674684. doi: 10.1016/j.ygeno.2005.08.004.

Huang, S. et al. (2010) 'The evolution of vertebrate tetraspanins: gene loss, retention, and massive positive selection after whole genome duplications.', BMC evolutionary biology, 10(1), p. 306. doi: 10.1186/1471-2148-10-306.

Kaji, K. et al. (2002) 'Infertility of CD9-Deficient Mouse Eggs Is Reversed by Mouse CD9, Human CD9, or Mouse CD81; Polyadenylated mRNA Injection Developed for Molecular Analysis of Sperm - Egg Fusion', 334, pp. 327-334. doi: 10.1006/dbio.2002.0694.

Knobeloch, K.-P. et al. (2000) 'Targeted Inactivation of the Tetraspanin CD37 Impairs T-CellDependent B-Cell Response under Suboptimal Costimulatory Conditions', Molecular and Cellular Biology, 20(15), pp. 5363-5369. doi: 10.1128/MCB.20.15.5363-5369.2000.

Leung, K. T. et al. (2011) 'The tetraspanin CD9 regulates migration, adhesion, and homing of human cord blood CD34+ hematopoietic stem and progenitor cells', Blood, 117(6), pp. 18401850. doi: 10.1182/blood-2010-04-281329.

Lin, Q. et al. (2019) 'Tracking genome-editing and associated molecular perturbations by SWATH mass spectrometry', Scientific Reports, 9(1), pp. 1-9. doi: 10.1038/s41598-01951612-z.

Metcalfe, W. K., Kimmel, C. B. and Schabtach, E. (1985) 'Anatomy of the posterior lateral line system in young larvae of the zebrafish', Journal of Comparative Neurology, 233(3), pp. 377389. doi: $10.1002 /$ cne. 902330307.

Meyer, A. and Van De Peer, Y. (2005) 'From 2R to 3R: Evidence for a fish-specific genome duplication (FSGD)', BioEssays. doi: 10.1002/bies.20293.

Minina, S., Reichman-Fried, M. and Raz, E. (2007) 'Control of Receptor Internalization, Signaling Level, and Precise Arrival at the Target in Guided Cell Migration', Current Biology, 17(13), pp. 1164-1172. doi: 10.1016/j.cub.2007.05.073.

Nusslein-Volhard, C. and R. D. (2002) 'Zebrafish (Practical Approach Series)', OUP Oxford 328. Olson, H. M. and Nechiporuk, A. V. (2018) 'Using zebrafish to study collective cell migration in development and disease', Frontiers in Cell and Developmental Biology. doi:

10.3389/fcell.2018.00083.

Stephens, B. and Handel, T. M. (2013) 'Chemokine receptor oligomerization and allostery', in Progress in Molecular Biology and Translational Science. doi: 10.1016/B978-0-12-3945877.00009-9.

Suárez, H. et al. (2021) 'CD9 inhibition reveals a functional connection of extracellular vesicle secretion with mitophagy in melanoma cells', Journal of Extracellular Vesicles, 10(7). doi: 10.1002/jev2.12082. 
Takeda, Y. et al. (2003) 'Tetraspanins CD9 and CD81 function to prevent the fusion of mononuclear phagocytes', Journal of Cell Biology, 161(5), pp. 945-956. doi: 10.1083/jcb.200212031.

Takeda, Y. et al. (2008) 'Double deficiency of tetraspanins CD9 and CD81 alters cell motility and protease production of macrophages and causes chronic obstructive pulmonary diseaselike phenotype in mice', Journal of Biological Chemistry. doi: 10.1074/jbc.M801902200.

Tarrant, J. M. et al. (2003) 'Tetraspanins: Molecular organisers of the leukocyte surface', Trends in Immunology, 24(11), pp. 610-617. doi: 10.1016/j.it.2003.09.011.

Thelen, M. and Legler, D. F. (2018) 'Membrane lipid environment: Potential modulation of chemokine receptor function', Cytokine. doi: 10.1016/j.cyto.2018.02.011.

Thisse, C. and Thisse, B. (2008) 'High-resolution in situ hybridization to whole-mount zebrafish embryos', Nature Protocols. doi: 10.1038/nprot.2007.514.

Trikic, M. Z. et al. (2011) 'Regulation of Zebrafish Hatching by Tetraspanin cd63', PLOS ONE, 6(5), p. e19683.

Valentin, G., Haas, P. and Gilmour, D. (2007) 'Report The Chemokine SDF1a Coordinates Tissue Migration through the Spatially Restricted Activation of Cxcr7 and Cxcr4b', pp. 10261031. doi: 10.1016/j.cub.2007.05.020.

Venkiteswaran, G. et al. (2014) 'Signaling Gradient Across a Migrating Tissue', 155(3). doi: 10.1016/j.cell.2013.09.046.Generation.

Wright, M. D. et al. (2004) 'Characterization of Mice Lacking the Tetraspanin Superfamily Member CD151 Characterization of Mice Lacking the Tetraspanin Superfamily Member CD151', Molecular and cellular biology, 24(13), pp. 5978-5988. doi: 10.1128/MCB.24.13.5978.

$\mathrm{Xu}, \mathrm{H}$. et al. (2014) 'GBeta1 controls collective cell migration by regulating the protrusive activity of leader cells in the posterior lateral line primordium', Developmental Biology, 385(2), pp. 316-327. doi: 10.1016/j.ydbio.2013.10.027.

Yáñez-Mó, M. et al. (2009) 'Tetraspanin-enriched microdomains: a functional unit in cell plasma membranes', Trends in Cell Biology, 19(9), pp. 434-446. doi:

10.1016/j.tcb.2009.06.004.

Yáñez-Mó, M., Gutiérrez-López, M. D. and Cabañas, C. (2011) 'Functional interplay between tetraspanins and proteases', Cellular and Molecular Life Sciences, 68(20), pp. 3323-3335. doi: 10.1007/s00018-011-0746-y. 
bioRxiv preprint doi: https://doi.org/10.1101/2021.05.19.444012; this version posted May 20, 2021. The copyright holder for this preprint (which was not certified by peer review) is the author/funder, who has granted bioRxiv a license to display the preprint in perpetuity. It is made available under aCC-BY 4.0 International license. 